\title{
BLOOD VOLUME DETERMINATIONS WITH RADIOACTIVE PHOSPHORUS
}

\author{
BY \\ GUSTAV NYLIN \\ From the Medical Clinic II, Sabbatsberg Hospital, Stockholm
}

Received December 3, 1944

In experiments on animals, Smith, Arnold, and Whipple (1921) compared the three then known methods for determining the circulating blood volume, namely, the Welcher method, the carbonic oxide method, and the dye method. They recommended the method worked out by Dawson, Evans, and Whipple (1920), the so-called " blue azo dye T 1824 " method, for determinations of plasma volume. Gibson and Evans (1937) modified this method and with their co-workers applied it to a comprehensive series of clinical problems. There is no doubt that, thanks to these activities, plasma volume determinations have been aided by valuable experiences, but for studies of the circulating blood volume every method used, even the last-mentioned, lacks to a certain extent the prerequisites needed for completely reliable values. For example, when determinations of the plasma volumes are made by the "blue azo dye" method, the dye substance introduced is not taken up solely by the circulating blood but, as Gibson himself admits, is also taken up by, inter alia, the reticulo-endothelial system. Cardozo (1940), who made a special study of the passing of T 1824 into the lymphatic system in dogs, comes to the following conclusion: "The passing of the dye to the lymph interferes with a correct interpretation of a disappearance curve according to Gibson and Evans, and it usually results in the calculation of too large plasma volumes." Between 15 to 30 minutes after the injection of the dye substance into the blood stream the lymph assumed a blue colour and after two hours reached a concentration up to 49 per cent of that in the plasma.

It has been shown in previous works (Nylin, 1944 and 1945), by employing the method of Hahn and Hevesy (1940 and 1942) for labelling the red blood corpuscles with radioactive phosphorus, that in normal cases mixing and equilibrium between the injected labelled blood corpuslces and the blood stream are attained remarkably early - a contrast to the dye method. Gibson showed that " mixing time" does not set in until after 15 minutes in the special case, and on an average after 7.5 minutes for 15 normal subjects. The continuation of the curve Gibson calls the " disappearance slope," and he states that only relative constancy is attained in that the curve in its further course falls slowly towards the abscissa. It seems as though Hevesy's method affords the possibility of studying a number of circulation problems that had not been possible previously, because the fundamental prerequisite appears to have been satisfied, when it is possible to inject the subject's own blood corpuscles labelled with $\mathbf{P}^{32}$ which are continuously confined to the circulating blood for a relatively long time, and whose activity comes into equilibrium with the circulating blood remarkably early. Hevesy, Warburg, et al. (1944) made determinations of the circulating blood volume in normals both by Hevesy's and Hahn's methods with radioactive phosphorus and by the $\mathrm{CO}$ method and also by the dye method. As was expected, with the labelled blood corpuscles they obtained lower and, very probably, more reliable values than by the other methods.

In the present work Hevesy's method has been employed on clinical cases, it being of importance by means of fractioned specimens, preferably from arterial blood, to obtain a knowledge of " the dilution curve," and particularly in determinations of the amount of blood in dilated hearts, where the amount of residual blood has proved to have a great influence on the type of curve (Nylin, 1945). Not until one knows the course of the individual dilution curve can one indicate more closely where mixing and equilibrium take place and subsequently select the points, preferably the mean value of several specimens, which are to be the basis for the calculation of the circulating blood volume.

\section{MethodS AND Results}

In the present work the same method has been employed as in those previously published by the writer. For determinations of the circulating blood volume the activity was established, by means 
of a Geiger counter, for the injected labelled blood corpuscles, and then for the fractioned specimens taken from the arterial blood. The calculation of the amount of circulating red blood corpuscles was made with the formula $x=\mathrm{A} \times \mathrm{B}$, where $x$ indicates the amount of circulating blood corpuscles required in c.c., $\mathrm{A}$, the amount of labelled blood corpuscles injected, and $\mathrm{B}$, the relation between the specific activity of the labelled blood corpuscles injected and the specific activity of the blood specimens taken. The specific activity of the latter constitutes the mean value for a series of specimens after equilibrium had been attained, each value including 3-18 separate determinations in a single case. With the guidance of the hematocrit and the amount of blood corpuscles, the amount of plasma was calculated. Finally, the total of the amounts of plasma and blood corpuscles gives the circulating blood volume. In calculating the amount of blood corpuscles expressed in grams a specific gravity of 1.08 , as used by Hevesy, has been taken.

The material comprises six subjects with normal hearts, six heart cases with varying ætiologies, and one case of anæmia; all were without signs of failure and had normal venous pressures. In addition there are two cases of typical cardiac failure, Case 14 with mitral stenosis and Case 15 with cardiosclerosis. The former had a venous pressure of $29 \mathrm{~cm}$., the latter one of $17 \mathrm{~cm}$. Both had much dilated hearts, as appears from their large heart volumes (see Table I).

For most cases detailed dilution curves have been published in an earlier work (Nylin, 1945). Among the normal cases, equilibrium between the injected activity and the circulating blood is attained already in the first minute in Case 1, and in the second and third minutes in the others. Case 2 was not subjected to a closer analysis of the dilution, but the specimens were taken as late as after the fifteenth minute. As emerges from Table I, the mean value for the amount of blood corpuscles for these seven normal cases is 1850 c.c., or $1998 \mathrm{~g}$. per kilogram of body weight: these normal cases have a weight of circulating blood corpuscles of $33.4 \mathrm{~g}$., somewhat less than that found by Hevesy et al., which was $36 \mathrm{~g}$. Nevertheless, the present material is so small that in this respect a comparison can hardly be made, but in each case the determination of the circulating blood volume is based, firstly, on a knowledge of the dilution curve in that individual case, and thus on a knowledge of when equilibrium was attained, and secondly on the mean value of a number of determinations on the same case.

In the group of compensated heart cases there is only one (Case 7), where equilibrium appears to be attained strikingly soon, in spite of the fact that the amount of residual blood in the heart would seem to be considerable. The heart volume is no less than 820 c.c. $/ \mathrm{m}^{2}{ }^{2}$ Repeated determinations with both decholin and with corpuscles labelled with radioactive phosphorus on the same patient showed remarkably short circulation times, which in this exceptional case could not be fully explained, though the possibility of a septal defect is not excluded. In the other cases in the last-mentioned group equilibrium set in later than in the normal cases, as appears from Table $\mathbf{I}$; this conforms with the course of the dilution curve and the large heart volumes, which has also been pointed out in an earlier work (Nylin; 1945).

The circulating blood corpuscle volume in compensated heart cases, expressed in c.c., grams, or grams per kilogram of body weight, and the total amount of circulating blood do not diverge from the normal value but exhibit striking agreement with them. In these heart cases, too, the blood volumes given for the individual case are the mean values of a number of observations.

Of particular interest is Case 8 , with a very large heart, 1230 c.c. $! \mathrm{m}^{2}$, where equilibrium appears to have been attained ten minutes after the injection of the labelled blood corpuscles. Four observations, made between 10 and 15 minutes after the injection, give a mean value of the amount of circulating blood corpuscles of 1275 c.c. The mean value of 8 observations 15-22 minutes after the injection is 1368 c.c. At the later determination an increase of $7 \cdot 3$ per cent in the circulating blood volume was obtained; that was probably due to the error of the method, which according to Hevesy et al. probably amounts to about 5 per cent. It is not out of the question that to some extent the increase may be due to the fact that as late as after the twenty-second minute, the activity had decreased somewhat and thus a somewhat too high figure for the amount of circulating blood corpuscles was indicated. We do not yet know with certainty how long the activity in the circulating blood remains constant.

The two with heart failure (Cases 14 and 15) have been subjected to detailed studies, and their dilution curves were published (Nylin, 1945). In spite of the considerable dilatation of the heart, probably with large amounts of residual blood, equilibrium nevertheless appears relatively early-from the second to the third minute. The amount of circulating blood corpuscles in Case 14 is as high as $46 \mathrm{~g}$. per kilogram, and in Case 15 is $36 \mathrm{~g}$. The total blood volume approaches twice the normal in Case 14, amounting to 6616 c.c. or 114 c.c. per kilogram of body weight. Remarkably enough Case 15 has neither so large an amount of blood corpuscles nor so great total blood volume, in spite of a pronounced insufficiency on May 15, 1944. Ten days later, when all the symptoms of failure had receded, the odema disappeared, and the body weight decreased by $12 \mathrm{~kg}$., both the 
BLOOD VOLUME

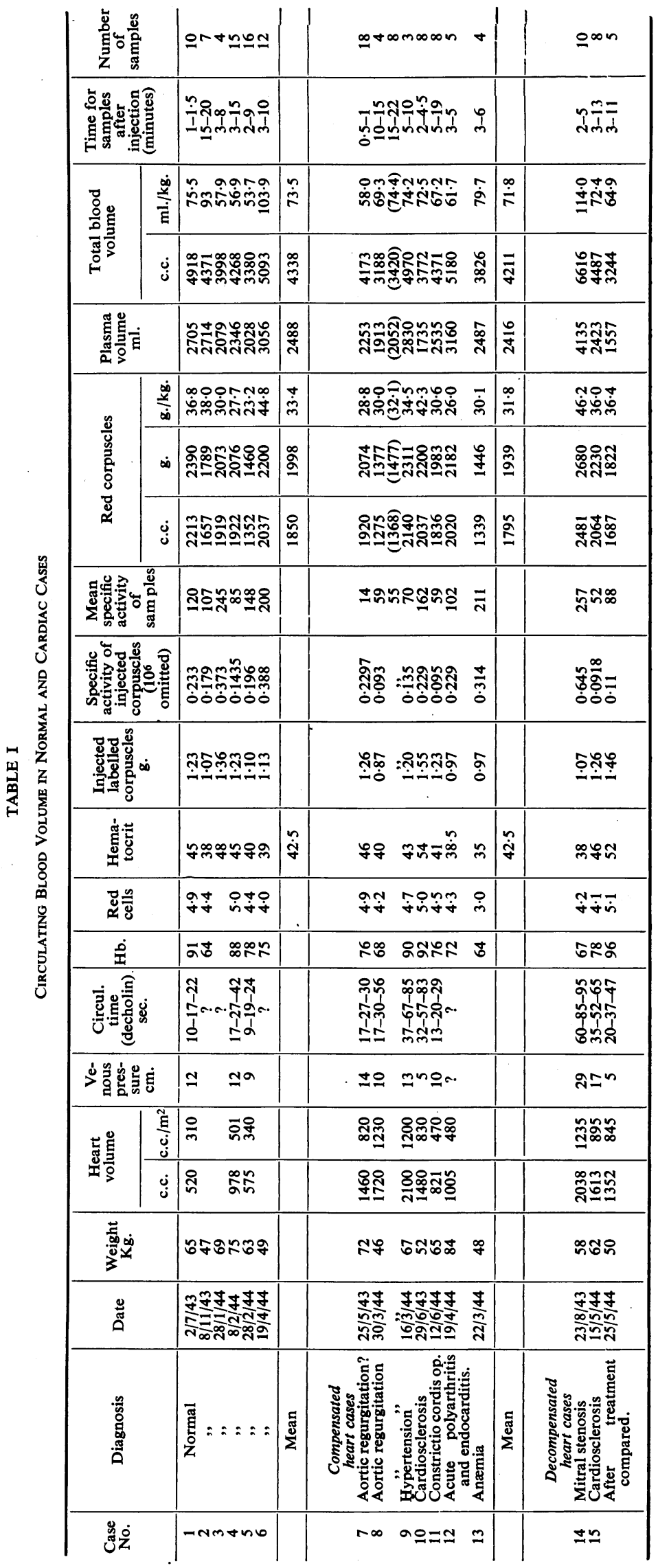


amount of blood corpuscles and the total blood volume had decreased, the latter by no less than 28 per cent, naturally owing to the disappearance of the fluid. It is remarkable on the other hand that the amount of blood corpuscles decreased by no less than 18 per cent-perhaps to accumulate in certain depots-in spite of the fact that both the hæmoglobin and the number of red blood corpuscles increased considerably.

\section{SUMMARY}

By the application of a new method worked out by Hevesy and his co-workers, employing blood corpuscles labelled with radioactive phosphorus, the circulating blood volume has been determined on both normal and cardiac cases, with and without failure. A prerequisate for the determination of the circulating blood volume is that the dilution curves can be established and thereby the time when equilibrium appears.

In this work the results in each case are based on the mean values of a number of determinations. The mean value for the amount of the circulating blood corpuscles in normal cases was found to be 1850 c.c., $1998 \mathrm{~g}$. or $33.4 \mathrm{~g}$. per kilogram of body weight. The mean value for the compensated cases is in agreement with the normal values at 1795 c.c., $1939 \mathrm{~g}$. and $31.8 \mathrm{~g}$. per kilogram of body weight. The total blood volume in the normal cases is 73.5 c.c. per kilogram of body weight, and in the compensated cases 71.8 c.c. Two cases of heart failure-and particularly one of them-show a considerable increase in both the amount of blood corpuscles and the circulating blood volume. The other case has been followed with repeated determinations after all signs of failure had disappeared, a great decrease in the circulating blood volume of no less than 28 per cent taking place simultaneously with the appearance of the interesting phenomenon that the amount of red blood corpuscles also decreased by 18 per cent.

I proffer my grateful thanks to Professor G. von Hevesy for all his kind advice, and I am greatly indebted to Professor M. Siegbahn for supplies of radioactive phosphorus.

This investigation has been made possible by a grant from the Therese and Johan Andersson Memorial Fund.

\section{REFERENCES}

Cardozo, L. E. (1940-41) Arch. Neerl. Physiol., 25, 410.

Dawson, A. B., Evans, H. M., and Whipple, G.'H. (1920). Amer. J. Physiol., 51, 232.

Gibson, J. G., and Evans, W. (1937). J. Clin. Invest., 16, 301.

Hahn, L., and Hevesy, G. (1949). Acta Physiol. Scand., 1, 3.

- (1942). Ibid., 4, 376.

Hevesy, G., Köster, K. H., Sørensen, G., Warburg, E., and Zerahn, K. (1944). Acta med. Scand., 116, 561.

Nylin, G., and Malm, M. (1944). Amer. J. med. Sci., 207, 743.

Nylin, G. (1945). Amer. Heart J. - -

Smith, H. P., Arnold, H. R., and Whipple, G. H. (1921). Amer. J. Physiol., 56, 337. 